\title{
EXTINCTION AND PERMANENCE OF TWO-NUTRIENT AND ONE-MICROORGANISM CHEMOSTAT MODEL WITH PULSED INPUT
}

XINYU SONG AND ZHONG ZHAO

Received 9 November 2005; Accepted 27 February 2006

A chemostat model with periodically pulsed input is considered. By using the Floquet theorem, we find that the microorganism eradication periodic solution $\left(u_{1}^{*}(t), v_{1}^{*}(t), 0\right)$ is globally asymptotically stable if the impulsive period $T$ is more than a critical value. At the same time we can find that the nutrient and microorganism are permanent if the impulsive period $T$ is less than the critical value.

Copyright (c) 2006 X. Song and Z. Zhao. This is an open access article distributed under the Creative Commons Attribution License, which permits unrestricted use, distribution, and reproduction in any medium, provided the original work is properly cited.

\section{Introduction}

A chemostat is a piece of laboratory apparatus frequently used for culturing microorganisms. It can be used for representing all kinds of microorganism systems such as lake, waste-water treatment and reaches for commercial production of the advantage of being easily implementable in a laboratory, and hence the model has been studied by more and more people. In its simplest form, the system approximates conditions for plankton growth in lakes, where the limiting nutrients such as silica and phosphate are supplied from streams draining the watershed. Chemostat with period inputs are studied in $[5,6,14]$, those with periodic washout rate in $[3,9]$ and those with periodic input and washout in [11]. We all know that nutrients are input into lakes and lakes are washed out when rain is falling. In fact, raining is not continuous. It occurs seasonally or in regular pulses. Thus, it is natural to describe this case in impulsive differential equations.

Impulsive differential equations are suitable for the mathematical simulation of evolutionary process in which the parameters undergo relatively long periods of smooth variation followed by a short-term rapid change (i.e., jumps) in their values. Recently, equations of this kind are found in almost every domain of applied science. Numerous examples are given in Bainov's and his collaborator's book [1,8]. Some impulsive differential equations have been recently introduced in population dynamics in relation to impulsive birth $[12,15]$, impulsive vaccination $[4,13]$, chemotherapeutic treatment of disease $[7,10]$, and population ecology [2]. 
In this paper, we consider the dynamics of two-nutrient and one-microorganism chemostat with pulsed input:

$$
\begin{gathered}
\dot{S}=-Q S-\frac{\mu_{1} x S}{\delta_{1}}, \\
\dot{R}=-R Q-\frac{\mu_{2} R x}{\delta_{2}}, \quad \bar{t} \neq n T, \\
\dot{x}=\mu_{1} x S+\mu_{2} x R-Q x, \\
\Delta S=S^{0} Q, \quad \Delta R=R^{0} Q, \quad \bar{t}=n T,
\end{gathered}
$$

where $S(t)$ and $R(t)$ denote the concentration of nutrient, and $x(t)$ denotes the concentration of the microorganism at time $t . S^{0}$ and $R^{0}$ represent that input concentration of the nutrient. $Q(0<Q<1)$ is referred to as the dilution rate. $\mu_{1}$ and $\mu_{2}$ denote the predation constants of predator, $\delta_{1}$ and $\delta_{2}$ show yield term, and $T$ is the period of pulse.

The aim of this work is to study the dynamical behaviors of two nutrients and one microorganism with pulsed input, and investigate how the impulsive perturbation affects the dynamical behaviors of unforced continuous system.

The variables in the above system may be rescaled by measuring $u=S / S^{0}, v=\delta_{2} /\left(\delta_{1} S^{0}\right) R$, $w=x / \delta_{1} S^{0}$, and $\bar{t}=Q t$, then we have the following system:

$$
\begin{gathered}
\dot{u}=-u-A u w \\
\dot{v}=-v-B v w, \quad t \neq n T, \\
\dot{w}=A u w+B v w-w, \\
\Delta u=Q, \quad \Delta v=p, \quad t=n T,
\end{gathered}
$$

where $p=\delta_{2} Q R^{0} / \delta_{1} S^{0}, A=\mu_{1} S^{0} / Q$, and $B=\mu_{2} \delta_{1} S^{0} / Q \delta_{2}$.

This paper is arranged as follows. In Section 2, we introduce some useful notations and definitions. In Section 3, by using Floquet theorem for the impulsive equation, smallamplitude perturbation skills and techniques of comparison, we get the local stability and global asymptotic stability of the microorganism eradication periodic solution. In Section 4, we show that the system is permanent if the impulsive period is less than some critical value. In Section 5, we give a brief discussion.

\section{Preliminaries}

In this section, we will give some definitions, notations, and some lemmas which will be useful for our main results.

Let $\mathbb{R}_{+}=[0, \infty), \mathbb{R}_{+}^{3}=\left\{x \in \mathbb{R}^{3}: x>0\right\}$. Denote $f=\left(f_{1}, f_{2}, f_{3}\right)$ the map defined by the right-hand side of the first three equations of system (1.2). Let $V: \mathbb{R}_{+} \times \mathbb{R}_{+}^{3} \longrightarrow \mathbb{R}_{+}$, then $V$ is said to belong to class $V_{0}$ if

(i) $V$ is continuous in $(n T,(n+1) T) \times \mathbb{R}_{+}^{3}$ and for each $x \in \mathbb{R}_{+}^{3}, n \in \mathbb{Z}_{+}$, $\lim _{(t, y) \rightarrow(n T, x)} V(t, y)=V\left(n T^{+}, x\right)$ exists;

(ii) $V$ is locally Lipschitzian in $x$. 
Definition 2.1. Let $V \in V_{0}$, then for $\left.(t, x) \in(n T,(n+1) T)\right] \times \mathbb{R}_{+}^{3}$, the upper-right derivative of $V(t, x)$ with respect to the impulsive differential system (1.2) is defined as

$$
D^{+} V(t, x)=\lim _{h \rightarrow 0^{+}} \sup \frac{1}{h}[V(t+h, x+f(t, x))-V(t, x)]
$$

Definition 2.2. System (1.2) is said to be permanent if there are constants $m>0, M>0$ and such that each positive solution $(u(t), v(t), w(t))$ of the system (1.2) satisfies $m \leq$ $u(t) \leq M, m \leq v(t) \leq M, m \leq w(t) \leq M$, for all $t \geq 0$. If all species of the system are permanent, then the system is called permanent.

Lemma 2.3. Suppose $\omega(t)=(u(t), v(t), w(t))$ is a solution of (1.2) subject to $\omega\left(0^{+}\right) \geq 0$ for all $t \geq 0$, and further suppose $\omega(t)>0, t \geq 0$ if $\omega\left(0^{+}\right)>0$.

Lemma 2.4. Let $V: \mathbb{R}_{+} \times \mathbb{R}^{3} \rightarrow \mathbb{R}_{+}$and $V \in V_{0}$. Assume that

$$
\begin{gathered}
D^{+} V(t, \omega) \leq g(t, V(t, \omega)), \quad t \neq n T, \\
V\left(t, \omega\left(t^{+}\right)\right) \leq \psi_{n}(V(t, \omega(t))), \quad t=n T,
\end{gathered}
$$

where $g: \mathbb{R}_{+} \times \mathbb{R}_{+} \rightarrow \mathbb{R}$ is continuous in $(n T,(n+1) T] \times \mathbb{R}_{+}$and for $y \in \mathbb{R}_{+}, n \in \mathbb{Z}_{+}$, $\lim _{(t, y) \rightarrow\left(n T^{+}, y\right)} g(t, y)$ exists, $\psi_{n}: \mathbb{R}^{+} \rightarrow \mathbb{R}^{+}$is nondecreasing. Let $r(t)$ be the maximal solution of the scalar impulsive differential equation

$$
\begin{gathered}
\frac{d u}{d t}=g(t, u), \quad t \neq n T, \\
u\left(t^{+}\right)=\psi_{n}\left(u_{n}(t)\right), \quad t=n T, \\
u\left(0^{+}\right)=u_{0}
\end{gathered}
$$

existing on $[0, \infty)$. Then $V\left(0^{+}, \omega_{0}\right) \leq u_{0}$ implies that $V(t, \omega(t)) \leq r(t), t \geq 0$, where $\omega(t)$ is any solution of system (1.2).

Consider the following system:

$$
\begin{aligned}
& \dot{u}=-u, \quad \dot{v}=-v, \quad t \neq n T, \\
& \Delta u=Q, \quad \Delta w=p, \quad t=n T .
\end{aligned}
$$

Lemma 2.5. System (2.4) has a positive periodic solution $\left(u^{*}(t), v^{*}(t)\right)$ and for every solution $(u(t), v(t))$ of (2.4), $\left|u(t)-u^{*}(t)\right| \rightarrow 0,\left|v(t)-v^{*}(t)\right| \rightarrow 0$ as $t \rightarrow \infty$, where $u^{*}(t)=$ $Q e^{-(t-n T)} /\left(1-e^{-T}\right), v^{*}(t)=p e^{-(t-n T)} /\left(1-e^{-T}\right)$. 
4 A two-nutrient and one-microorganism chemostat model

\section{Extinction}

In this section, we study the stability of the microorganism-free periodic solution as a solution of the full system (1.2). Firstly, we present the Floquet theory for the linear $T$ periodic impulsive equation:

$$
\begin{gathered}
\frac{d x}{d t}=A(t) x, \quad t \neq \tau_{k}, t \in \mathbb{R}, \\
\Delta x=B_{k} x, \quad t=\Delta_{k}, \quad k \in \mathbb{Z} .
\end{gathered}
$$

Then we introduce the following conditions:

$\left(\mathrm{H}_{1}\right) A(\cdot) \in P C\left(R, C^{n \times n}\right)$ and $A(t+T)=A(t)(t \in \mathbb{R})$,

$\left(\mathrm{H}_{2}\right) B \in C^{n \times n} \cdot \operatorname{det}\left(E+B_{k}\right) \neq 0, \tau_{k}<\tau_{k+1}(k \in \mathbb{Z})$

$\left(\mathrm{H}_{3}\right)$ there exists a $q \in \mathbb{N}$ such that

$$
B_{k+q}=B_{k}, \quad \tau_{k+q}=\tau_{k}+T, \quad k \in \mathbb{Z} .
$$

Let $\Phi(t)$ be a fundamental matrix of (3.1), then there exists a unique nonsingular matrix $M \in \mathbb{C}^{n \times n}$ such that

$$
\Phi(t+T)=\Phi(t) M, \quad t \in \mathbb{R}
$$

By equality (3.3) there corresponds to the fundamental matrix $\Phi(t)$ the constant matrix $M$ which we call the monodromy matrix of (3.1) (corresponding to the fundamental matrix of $\Phi(t))$.

All monodromy matrices of (3.1) are similar and have the same eigenvalues. The eigenvalues $\mu_{1}, \ldots, \mu_{n}$ of the monodromy matrices are called the Floquet multipliers of (3.1).

Lemma 3.1 (Floquet theory). Let conditions $\left(H_{1}\right)-\left(H_{3}\right)$ hold. Then the linear T-periodic impulsive (3.1) is

(1) stable if and only if all multipliers $\mu_{j}(j=1, \ldots, n)$ of (3.1) satisfy the inequality $\left|\mu_{j}\right| \leq 1$ and, moreover, to those $\mu_{j}$ for which $\left|\mu_{j}\right|=1$, there correspond simple elementary divisors;

(2) asymptotically stable if and only if all monodromy $\mu_{j}(j=1, \ldots, n)$ of $(3.1)$ satisfy the inequality $\left|\mu_{j}\right|<1$;

(3) unstable if $\left|\mu_{j}\right|>1$ for some $j=1, \ldots, n$.

Theorem 3.2. Let $\omega(t)=(u(t), v(t), w(t))$ be any solution of system (1.2), then $\left(u^{*}(t)\right.$, $\left.v^{*}(t), 0\right)$ is globally asymptotically stable, provided that $T>A Q+B p$.

Proof. Firstly, we prove the locally stable. The local stability of the periodic solution $\left(u^{*}(t), v^{*}(t), 0\right)$ may be determined by considering the behavior of small-amplitude perturbations of the solution. Define $u(t)=u^{*}(t)+x_{1}(t), v(t)=v^{*}(t)+x_{2}(t), w(t)=w^{*}(t)+$ $x_{3}(t)$, where $x_{1}, x_{2}$, and $x_{3}$ are small perturbations. Equation (1.2) can be expanded in a 
Taylor series: after neglecting higher-order terms, the linearized equations are read as

$$
\begin{gathered}
\dot{x}_{1}=-x_{1}-A u^{*}(t) x_{3}, \\
\dot{x}_{2}=-x_{2}-B v^{*}(t) x_{3}, \\
\dot{x}_{3}=\left(A u^{*}(t)+B v^{*}(t)-1\right) x_{3} .
\end{gathered}
$$

Let $\Phi(t)$ be the fundament matrix of (3.4), then $\Phi(t)$ must satisfy

$$
\frac{d \Phi(t)}{d t}=\left(\begin{array}{ccc}
-1 & 0 & -A u^{*}(t) \\
0 & -1 & -B v^{*}(t) \\
0 & 0 & A u^{*}(t)+B v^{*}(t)
\end{array}\right) \Phi(t)
$$

Thus, linearization of (1.2) from the fourth to the sixth becomes

$$
\left(\begin{array}{l}
x_{1}\left(n T^{+}\right) \\
x_{2}\left(n T^{+}\right) \\
x_{3}\left(n T^{+}\right)
\end{array}\right)=\left(\begin{array}{lll}
1 & 0 & 0 \\
0 & 1 & 0 \\
0 & 0 & 1
\end{array}\right)\left(\begin{array}{l}
x_{1}(n T) \\
x_{2}(n T) \\
x_{3}(n T)
\end{array}\right)
$$

Thus, the monodromy matrix of (3.4) is

$$
M=\left(\begin{array}{lll}
1 & 0 & 0 \\
0 & 1 & 0 \\
0 & 0 & 1
\end{array}\right)=\Phi(T)
$$

From (3.5) we have $\Phi(T)=\Phi(0) \exp \left(\int_{0}^{T} A d t\right) \triangleq \Phi(0) \exp (\bar{A})$, where $\Phi(0)$ is the identity matrix. Let $\lambda_{1}, \lambda_{2}$, and $\lambda_{3}$ be eigenvalues of matrix $\bar{A}$, then $\lambda_{1}<0, \lambda_{2}<0, \lambda_{3}=A Q+$ $B p-T<0$. Therefore, all eigenvalues of $M$, namely, $e^{\lambda_{1}}, e^{\lambda_{2}}$, and $e^{\lambda_{3}}$ have absolute values less than one if and only if $T>A Q+B p$. According to Floquet theorem (Lemma 3.1), the $\left(u^{*}(t), v^{*}(t), 0\right)$ is locally stable.

In the following, we prove the global attractivity. Choose $\varepsilon_{1}>0$ and $\varepsilon_{2}>0$ such that

$$
\delta=A Q+\varepsilon_{1} T+B p+\varepsilon_{2} T-T<0 .
$$

Noting that $\dot{u} \leq-u, \dot{v} \leq-v$ and considering the following impulsive differential equations:

$$
\begin{gathered}
\dot{y}_{1}(t)=-y_{1}(t), \quad t \neq n T, \\
\Delta y_{1}=Q, \quad t=n T, \\
\dot{y}_{2}(t)=-y_{2}(t), \quad t \neq n T, \\
\Delta y_{2}=p, \quad t=n T,
\end{gathered}
$$


6 A two-nutrient and one-microorganism chemostat model

we have $u(t) \leq y_{1}(t), v(t) \leq y_{2}(t)$, and $y_{1}(t) \rightarrow y_{1}^{*}(t), y_{2}(t) \rightarrow y_{2}^{*}(t)$ as $t \rightarrow \infty$, where $y_{1}^{*}(t), y_{2}^{*}(t)$ is the periodic solution. Then

$$
\begin{aligned}
& u(t) \leq y_{1}(t)<y_{1}^{*}(t)+\varepsilon_{1}, \\
& v(t) \leq y_{2}(t)<y_{2}^{*}(t)+\varepsilon_{2},
\end{aligned}
$$

for $t$ large enough. For simplification, we may assume (3.10) holds for all $t \geq 0$. From (1.2), we can get

$$
\dot{w} \leq w\left(A\left(u^{*}(t)+\varepsilon_{1}\right)+B\left(v^{*}(t)+\varepsilon_{2}\right)-1\right),
$$

integrate $(3.11)$ on $(n T,(n+1) T]$ which yields

$$
w(n+1) T \leq w\left(n T^{+}\right) \exp \int_{n T}^{(n+1) T}\left[A\left(u^{*}(t)+\varepsilon_{1}\right)+B\left(v^{*}(t)+\varepsilon_{2}\right)-1\right] d t,
$$

therefore, we have $w(n+1) T \leq w\left(n T^{+}\right) \exp (\delta)$. Thus $w(n T) \leq w\left(0^{+}\right) \exp (n \delta)$ and $w(n T) \rightarrow$ 0 as $n \rightarrow \infty$, since $0<w(t)<w(n T)$. Therefore $w(t) \rightarrow 0$ as $t \rightarrow \infty$.

Next we prove that $u(t) \rightarrow u^{*}(t), v(t) \rightarrow v^{*}(t)$ as $t \rightarrow \infty$, if $\lim _{t \rightarrow \infty} w(t)=0$. For $0<\varepsilon_{3}<$ $\min \{1 / A, 1 / B\}$, there exists a $\widehat{T}>0$ such that $0<w(t)<\varepsilon_{3}$, for all $t \geq \widehat{T}$. Then we have

$$
-u\left(1+A \varepsilon_{3}\right) \leq \dot{u} \leq-u
$$

By Lemma 2.4 we obtain $y_{3}(t) \leq u(t) \leq y_{1}(t)$, and $y_{1}(t) \rightarrow u^{*}(t)$ and $y_{3}(t) \rightarrow y_{3}^{*}(t)$ as $t \rightarrow \infty$, where $y_{3}(t)$ is the solution of

$$
\begin{gathered}
\dot{y}_{3}=-y_{3}\left(1+B \varepsilon_{3}\right), \quad t \neq n T, \\
\Delta y_{3}=Q, \quad t=n T,
\end{gathered}
$$

and $y_{3}^{*}(t)=Q \exp \left(-1-B \varepsilon_{3}\right)(t-n T) /\left(1-\exp \left(-1-\varepsilon_{3} B\right) T\right), n T<t \leq(n+1) T$, therefore $y_{3}^{*}(t)-\varepsilon_{4}<u(t)<u^{*}(t)+\varepsilon_{4}, \varepsilon_{4}>0$, for $t$ large enough. Let $\varepsilon_{3} \rightarrow 0$, we get $y_{3}^{*}(t) \rightarrow$ $u^{*}(t)$. Hence $u(t) \rightarrow u^{*}(t)$ as $t \rightarrow \infty$. By the same method, we can prove $v(t) \rightarrow v^{*}(t)$, as $t \rightarrow \infty$. This completes the proof.

\section{Permanence}

First, we show that all solutions of (1.2) are uniformly ultimately bounded.

Theorem 4.1. There exists a constant $M>0$ such that $u(t) \leq M, v(t) \leq M, w(t) \leq M$ for each positive solution $\omega(t)=(u(t), v(t), w(t))$ of (1.2) with $t$ large enough.

Proof. Define a function $V(t, \omega(t))=u+v+w$, then $V(t, \omega(t)) \in V_{0}$ and the upper-right derivative of $V(t, \omega(t))$ along a solution of (1.2) is described as

$$
\begin{gathered}
D^{+} V(t, \omega(t)) \leq-V, \quad t \neq n T, \\
\Delta V=p+Q, \quad t=n T,
\end{gathered}
$$


we obtain

$$
V(t) \leq V\left(0^{+}\right) e^{-t}+(p+Q) \frac{e^{-(t-T)}}{1-e^{-T}}+\frac{(p+Q) e^{T}}{e^{T}-1} \longrightarrow \frac{(p+Q) e^{T}}{e^{T}-1}, \quad t \longrightarrow \infty .
$$

By the definition of $V(t, \omega(t))$, we obtain that each positive solution of (1.2) is uniformly ultimately bounded.

Next we give the conditions of permanence.

Theorem 4.2. System (1.2) is permanent if $T<A Q+B p$.

Proof. Suppose $\omega(t)=(u(t), v(t), w(t))$ is a solution of (1.2) with positive initial value. From Theorem 4.1 we may assume $u(t) \leq M, v(t) \leq M, w(t) \leq M, t \geq 0$, and $M>0$. From system (1.2), let $m_{1}=Q e^{-T} /(1-e-T)-\varepsilon_{5}, \varepsilon_{5}>0$, and $m_{2}=p e^{-T} /\left(1-e^{-T}\right)-\varepsilon_{6}$, $\varepsilon_{6}>0$. According to Lemma 2.4, we have $u(t) \geq m_{1}$ and $v(t) \geq m_{2}$ for $t$ large enough.

In the following, we want to find $m_{3}$ such that $w(t) \geq m_{3}$ for $t$ large enough. We will do it in the following two steps for convenience.

Step 1. Let $m_{3}>0$ and $\varepsilon_{7}, \varepsilon_{8}$ be small enough, such that

$$
\rho=A Q+B p-\varepsilon_{7} T-\varepsilon_{8} T-T>0 .
$$

We will prove $w(t)<m_{3}$ cannot hold for all $t \geq 0$. Otherwise,

$$
\frac{d u}{d t} \geq-\left(1+A m_{3}\right) u
$$

from Lemma 2.4, we have $u(t) \geq z(t)$ and $z(t) \rightarrow z^{*}(t), t \rightarrow \infty$, where $z(t)$ is the solution of

$$
\begin{gathered}
\frac{d z}{d t}=-\left(1+A m_{3}\right) z, \quad t \neq n T, \\
\Delta z=Q, \quad t=n T, \\
z^{*}(t)=\frac{Q \exp \left(-1-A m_{3}\right)(t-n T)}{1-\exp \left(-1-A m_{3}\right) T}, \quad t \in(n T,(n+1) T] .
\end{gathered}
$$

Therefore, there exists a $n_{1}>0, t>n_{1} T$ such that $w(t) \geq z(t)>z^{*}(t)-\varepsilon_{7}$ at the same time, we have

$$
v(t) \geq-\left(1+B m_{3}\right) v(t)
$$

similarly, we have $v(t) \geq z_{1}(t), z_{1}(t) \rightarrow z_{1}^{*}(t)(t \rightarrow \infty)$, where $z_{1}^{*}(t)$ is the solution of

$$
\begin{gathered}
\dot{z}_{1}(t)=-\left(1+B m_{3}\right) z_{1}, \quad t \neq n T \\
\Delta z_{1}=p, \quad t=n T
\end{gathered}
$$


8 A two-nutrient and one-microorganism chemostat model

and $z_{1}^{*}(t)=p \exp \left(-1-B m_{3}\right)(t-n T) /\left(1-\exp \left(-1-B m_{3}\right) T\right), t \in(n T,(n+1) T]$. For $n>$ $n_{2}>n_{1}$, we have

$$
\frac{d w}{d t} \geq\left[A\left(z^{*}(t)-\varepsilon_{7}\right)+B\left(z_{1}^{*}(t)-\varepsilon_{8}\right)-1\right] w .
$$

Integrating (4.8) on $t \in(n T,(n+1) T], n>n_{2}$, we obtain

$$
w(n+1) T \geq w(n T) \exp \int_{n T}^{(n+1) T}\left[A\left(z^{*}(t)-\varepsilon_{7}\right)+B\left(z_{1}^{*}(t)-\varepsilon_{8}\right)-1\right] d t
$$

therefore,

$$
w((n+1) T) \geq w(n T) \exp (\rho)
$$

Then $w\left(N_{1}+k\right) T \geq w\left(N_{1} T\right) \exp (k \rho) \rightarrow \infty$ as $t \rightarrow \infty$, which is a contradiction to the boundedness of $w(t)$.

Step 2. If $w(t) \geq m_{3}$ for all $t>t_{1}$, then our aim is obtained. Otherwise, $w(t) \leq m_{3}$ for some $t \geq t_{1}$. Setting $t^{*}=\inf _{t>t^{*}}\left\{w(t)<m_{3}\right\}$, then we have $w(t) \geq m_{3}$ for $t \in\left[t, t^{*}\right)$, and $w\left(t^{*}\right)=m_{3}$. Since $w(t)$ is continuous, suppose $t^{*} \in\left(n_{1} T,\left(n_{1}+1\right) T\right], n_{1} \in \mathbb{N}$, select $n_{2} \in$ $\mathbb{N}, n_{3} \in \mathbb{N}$, such that

$$
\begin{gathered}
n_{2} T>\frac{1}{-1-A m_{3}} \ln \frac{\varepsilon_{7}}{M+Q}, \\
n_{2} T>\frac{1}{-1-B m_{3}} \ln \frac{\varepsilon_{8}}{M+p}, \\
\exp \left(\eta\left(n_{2}+1\right) T\right) \exp \left(n_{3} \rho\right)>1,
\end{gathered}
$$

where $\eta=-1+A m_{1}+B m_{2}<0$. Let $T^{\prime}=n_{2} T+n_{3} T$, we claim that there must exist a $t^{\prime} \in$ $\left(\left(n_{1}+1\right) T,\left(n_{1}+1\right) T+T^{\prime}\right]$ such that $w(t) \geq m_{3}$; otherwise $w(t)<m_{3}, t \in\left(\left(n_{1}+1\right) T,\left(n_{1}+\right.\right.$ 1) $\left.T+T^{\prime}\right]$. Consider (4.5) and (4.7) with $z\left(\left(n_{1}+1\right) T^{+}\right)=u\left(\left(n_{1}+1\right) T^{+}\right), z_{1}\left(\left(n_{1}+1\right) T^{+}\right)=$ $v\left(\left(n_{1}+1\right) T^{+}\right)$, we have

$$
\begin{gathered}
z(t)=\left(z\left(\left(n_{1}+1\right) T^{+}\right)-\frac{Q}{1-e^{-\left(1+A m_{3}\right) T}}\right) e^{-\left(1+A m_{3}\right)\left(t-\left(n_{1}+1\right) T\right)}+z^{*}(t), \\
z_{1}(t)=\left(z_{1}\left(\left(n_{1}+1\right) T^{+}\right)-\frac{p}{1-e^{-\left(1+B m_{3}\right) T}}\right) e^{-\left(1+B m_{3}\right)\left(t-\left(n_{1}+1\right) T\right)}+z_{1}^{*}(t),
\end{gathered}
$$

for $t \in(n T,(n+1) T], n_{1}+1<n \leq n_{1}+n_{2}+n_{3}+1$. Then

$$
\begin{gathered}
\left|z(t)-z^{*}(t)\right| \leq(M+Q) e^{-\left(1+A m_{3}\right)\left(t-\left(n_{1}+1\right) T\right)}<\varepsilon_{7}, \\
\left|z_{1}(t)-z_{1}^{*}(t)\right| \leq(M+p) e^{-\left(1+B m_{3}\right)\left(t-\left(n_{1}+1\right) T\right)}<\varepsilon_{8},
\end{gathered}
$$

and $u(t) \geq z(t)>z^{*}(t)-\varepsilon_{7}, v(t) \geq z_{1}(t)>z_{1}^{*}(t)-\varepsilon_{8}$, for $\left(n_{1}+1+n_{2}\right) T \leq t \leq\left(n_{1}+1\right) T+$ $T^{\prime}$, which implies (4.8) holds. For $\left(n_{1}+1+n_{2}\right) T \leq t \leq\left(n_{1}+1\right) T+T^{\prime}$, as in Step 1, we 
have $w\left(\left(n_{1}+n_{2}+n_{3}+1\right) T\right) \geq w\left(\left(n_{1}+n_{2}+1\right) T\right) \exp \left(n_{3} \rho\right)$; there are two possible cases for $t \in\left(t^{*},\left(n_{1}+1\right) T\right]$.

Case 1. If $w(t)<m_{3}$ for $t \in\left(t^{*},\left(n_{1}+1\right) T\right]$, then $w(t)<m_{3}$ for all $t \in\left(t^{*},\left(n_{1}+1+n_{2}\right) T\right]$; system (1.2) gives

$$
\dot{w}(t) \geq w(t)\left(-1+A m_{1}+B m_{2}\right)=\eta w(t),
$$

integrating (4.15) on $\left(t^{*},\left(n_{1}+1+n_{2}\right) T\right]$, which yields $w\left(\left(n_{1}+n_{2}+1\right) T\right) \geq m_{3} \exp \left(\eta\left(n_{2}+\right.\right.$ $1))$, then $w\left(n_{1}+1+n_{2}+n_{3}\right) T \geq m_{3} \exp \left(\eta\left(n_{2}+1\right) T\right) \exp \left(n_{3} \rho\right)>m_{3}$, which is a contraction. Let $\bar{t}=\inf _{t>t^{*}}\left\{w(t) \geq m_{3}\right\}$, then $w(\bar{t})=m_{3}$ and (4.15) holds for $t \in\left[t^{*}, \bar{t}\right)$. Then integrating (4.15) on $\left[t^{*}, \bar{t}\right)$ yields $w(t) \geq w\left(t^{*}\right) \exp \left(\eta\left(t-t^{*}\right)\right) \geq m_{3} \exp \left(\eta\left(1+n_{2}+n_{3}\right) T\right) \triangleq$ $\bar{m}_{3}$. For $t>\bar{t}$, the same argument can be continued. Since $w(\bar{t}) \geq m_{3}$, hence $w(t) \geq \bar{m}_{3}$ for all $t>t_{1}$.

Case 2. There exists a $t^{\prime} \in\left(t^{*},\left(n_{1}+1\right) T\right]$ such that $w\left(t^{\prime}\right) \geq m_{3}$. Let $\hat{t}=\inf _{t>t^{*}}\{w(t) \geq$ $\left.m_{3}\right\}$, then $w(t)<m_{3}$ for $t \in\left[t^{*}, \hat{t}\right)$ and $w(\hat{t})=m_{3}$ for $t \in\left[t^{*}, \hat{t}\right),(4.15)$ holds, and integrating (4.15) on $\left[t^{*}, \hat{t}\right)$, we have $w(t) \geq w\left(t^{*}\right) \exp \left(\eta\left(t-t^{*}\right)\right) \geq m_{3} \exp (\eta T)>\bar{m}_{3}$. This process can be continued since $w(\hat{t}) \geq m_{3}$, and we have $w(t) \geq \bar{m}_{1}$ for $t>t_{1}$. Thus in both cases, we conclude $w(t) \geq \bar{m}_{3}$ for all $t>t_{1}$.

Incorporating into Theorem 4.1 , the proof is complete.

\section{Discussion}

In this paper, we have investigated the model for a chemostat with two nutrients and one microorganism and periodically pulsed substrate. We have proved that microorganism eradication periodic solution $\left(u^{*}(t), v^{*}(t), 0\right)$ is globally asymptotically stable if $T>A Q+$ $B p$, which is showed in Figure 5.1. We can see that the variables $u(t), v(t)$ oscillate in a stable periodical cycle, in contrast with $w(t)$ rapidly decrease to zero. At the same time we also have proved the system (1.2) is permanent if $T<A Q+B p$, which is simulated in Figure 5.2. The variables $u(t), v(t), w(t)$ oscillate in a stable periodical cycle, respectively. So we can find that $T=A Q+B p$ is a threshold. In fact, when the period of pulses is less than the threshold, the nutrients and microorganism coexist. If the period is more than the threshold, the microorganism will become extinct.

If we replace the pulse input in system (1.2) with continuous input, the system (1.2) becomes

$$
\begin{gathered}
\dot{u}=\frac{Q}{T}-u-A u w, \\
\dot{v}=\frac{p}{T}-v-B v w, \\
\dot{w}=A u w+B v w-w,
\end{gathered}
$$

there also exists a microorganism eradication equilibrium for system $(5.1)$, that is, $(Q / T$, $p / T, 0)$, which is globally asymptotically stable if $T>A Q+B p$, see the appendix. The result is the same as our system (1.2), the result is simulated in Figure 5.3. We can obtain impulsive input effect that is the same as the continuous input. 
10 A two-nutrient and one-microorganism chemostat model

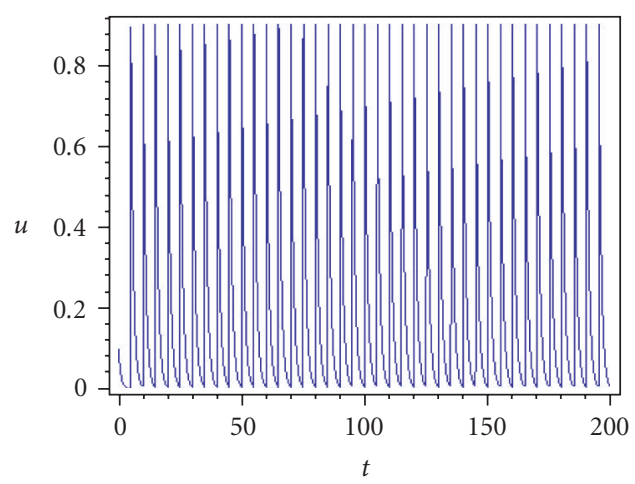

(a)

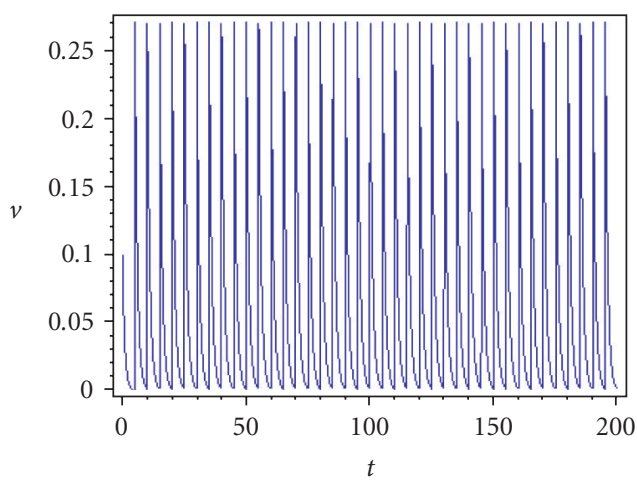

(b)

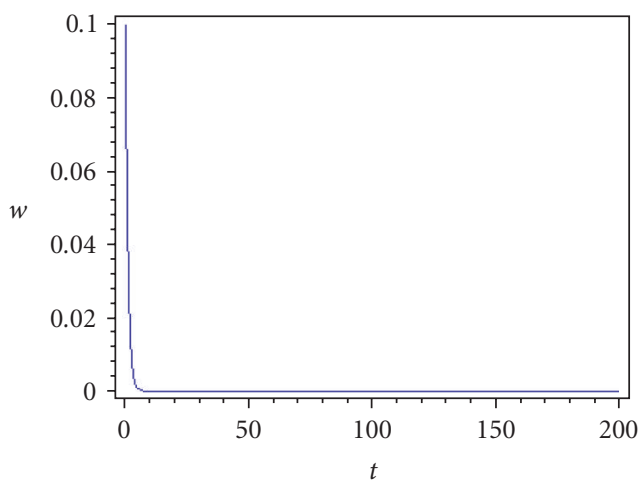

(c)

Figure 5.1. Time series of the system (3.1) with pulse, $A=5 / 9, p=0.54, B=20 / 9, Q=0.9$, and $T=5$. 


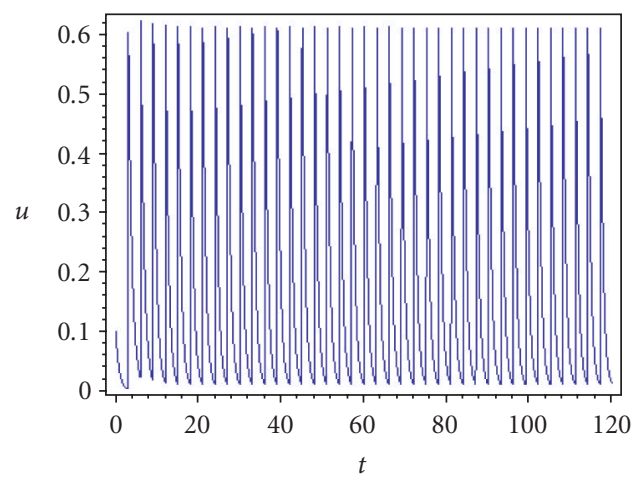

(a)

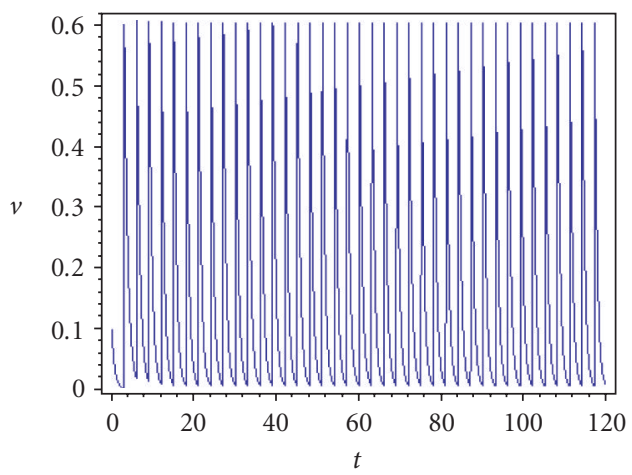

(b)

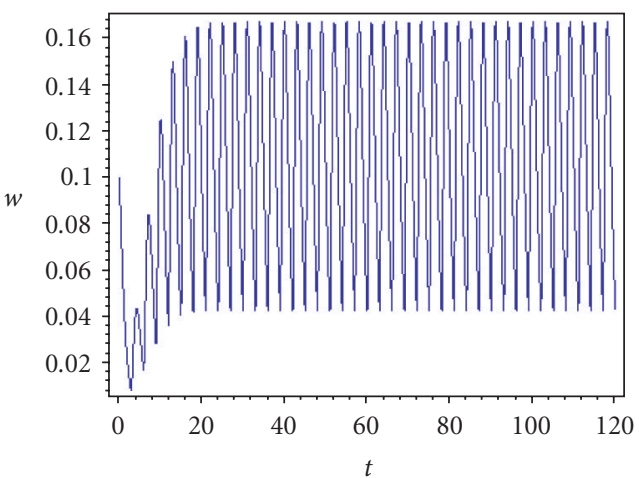

(c)

Figure 5.2. Time series of the system (3.1) with pulse, $A=5, B=20 / 3, p=0.6, Q=0.6$, and $T=3$. 
12 A two-nutrient and one-microorganism chemostat model

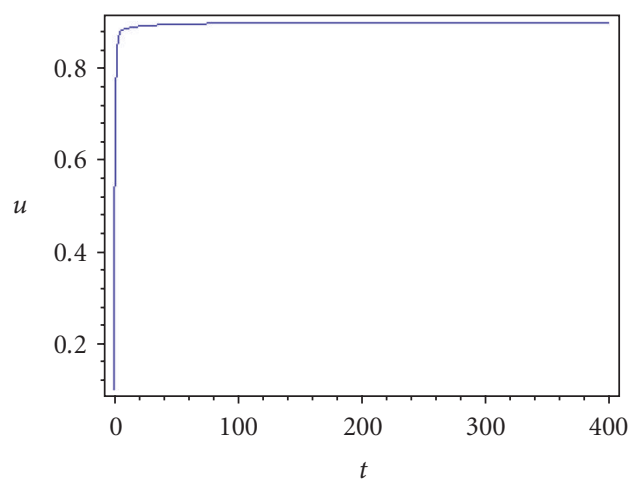

(a)

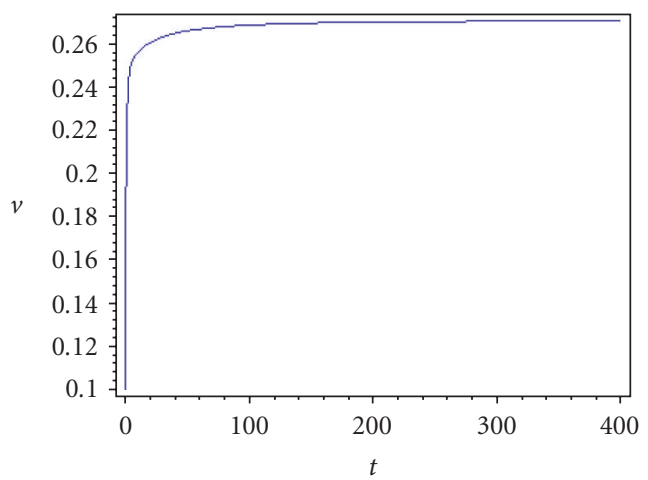

(b)

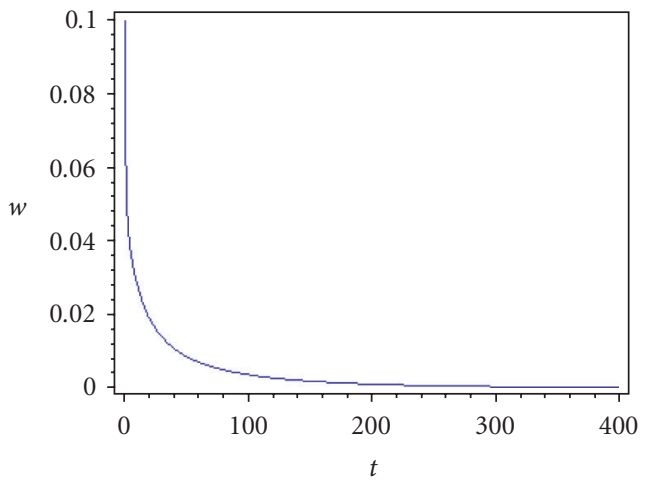

(c)

Figure 5.3. Time series of the system (3.1) without pulse, $A=5 / 9, B=20 / 9, p=0.54, Q=0.9$, and $T=10$. 


\section{Appendix}

In this appendix, we will prove that the microorganism eradication equilibrium $(\tilde{u}, \tilde{v}, 0)$ is globally stable with respect to int $\mathbb{R}_{+}^{3}$ if $T>A Q+B p$, where $\tilde{u}=Q / T, \tilde{v}=p / T, \mathbb{R}_{+}^{3}=$ $\{(u, v, w):, u>0, v>0, w>0\}$.

Let us denote the Jacobian matrix system (5.1) evaluated at the equilibrium point $E(Q / T, p / T, 0)$ by $J(E)$. Then

$$
\left(\begin{array}{ccc}
-1 & 0 & -\frac{A Q}{T} \\
0 & -1 & -\frac{B p}{T} \\
0 & 0 & \frac{A Q}{T}+\frac{B p}{T}-1
\end{array}\right)
$$

It is easy to see that all eigenvalues of $J(E)$ are negative if and only if $T>A Q+B p$. Therefore, $E(Q / T, p / T, 0)$ is locally stable if $T>A Q+B p$. Let $V(u, v, w)$ be positive definite function about $(u, v, w)$ given by

$$
V(u, v, w)=c_{1}\left(u-\tilde{u}-\tilde{u} \ln \frac{u}{\tilde{u}}\right)+c_{2}\left(v-\tilde{v}-\tilde{v} \ln \frac{v}{\widetilde{v}}\right)+c_{3} w
$$

where $c_{i}>0, i=1,2,3$. Then the derivative of $V$ along with the solution of system (5.1) is $\dot{V}=-c_{1}(u-\tilde{u})^{2} / u-c_{2}(v-\tilde{v})^{2} / v+A\left(c_{3}-c_{1}\right) w(u-\tilde{u})+B\left(c_{3}-c_{2}\right) w(v-\tilde{v})+c_{3}(A Q / T+$ $B p / T-1) w$, we choose $c_{i}(i=1,2,3)$, such that $c_{1}=c_{2}=c_{3}$. Then $V$ is negative definite in int $\mathbb{R}_{+}^{3}$ if and only if $T>A Q+B p$. So the microorganism eradication equilibrium $(\tilde{u}, \tilde{v}, 0)$ is globally stable in int $\mathbb{R}_{+}^{3}$.

\section{Acknowledgments}

This work is supported by the National Natural Science Foundation of China (no. 10471117), the Henan Innovation Project for University Prominent Research Talents (no. 2005KYCX017), and the Scientific Research Foundation for the Returned Overseas Chinese Scholars, State Education Ministry.

\section{References}

[1] D. D. Bainnov and D. D. Simeonov, Impulsive Differential Equations: Periodic Solutions and Applications, Pitman Monographs and Surveys in Pure and Applied Mathematics, vol. 66, Longman Scientific \& Technical, Harlow, 1993.

[2] G. Ballinger and X. Liu, Permanence of population growth models with impulsive effects, Mathematical and Computer Modelling 26 (1997), no. 12, 59-72.

[3] G. J. Butler, S. B. Hsu, and P. Waltman, A mathematical model of the chemostat with periodic washout rate, SIAM Journal on Applied Mathematics 45 (1985), no. 3, 435-449.

[4] A. D'Onofrio, Stability properties of pulse vaccination strategy in SEIR epidemic model, Mathematical Biosciences 179 (2002), no. 1, 57-72.

[5] J. K. Hale and A. S. Somolinos, Competition for fluctuating nutrient, Journal of Mathematical Biology 18 (1983), no. 3, 255-280. 


\section{A two-nutrient and one-microorganism chemostat model}

[6] S. B. Hsu, A competition model for a seasonally fluctuating nutrient, Journal of Mathematical Biology 9 (1980), no. 2, 115-132.

[7] A. Lakmeche and O. Arino, Bifurcation of non trivial periodic solutions of impulsive differential equations arising chemotherapeutic treatment, Dynamics of Continuous, Discrete and Impulsive Systems 7 (2000), no. 2, 265-287.

[8] V. Lakshmikantham, D. D. Baĭnov, and P. S. Simeonov, Theory of Impulsive Differential Equations, Series in Modern Applied Mathematics, vol. 6, World Scientific, New Jersey, 1989.

[9] P. Lenas and S. Pavlous, Coexistence of three competing microbial populations in a chemostat with periodically varying dilution rate, Mathematical Biosciences 129 (1995), no. 2, 111-142.

[10] J. C. Panetta, A mathematical model of periodically pulsed chemotherapy: tumor recurrence and metastasis in a competitive environment, Bulletin of Mathematical Biology 58 (1996), no. 3, 425447.

[11] S. S. Pilyugin and P. Waltman, Competition in the unstirred chemostat with periodic input and washout, SIAM Journal on Applied Mathematics 59 (1999), no. 4, 1157-1177.

[12] M. G. Roberts and R. R. Kao, The dynamics of an infectious disease in a population with birth pulses, Mathematical Biosciences 149 (1998), no. 1, 23-36.

[13] B. Shulgin, L. Stone, and Z. Agur, Pulse vaccination strategy in the SIR epidemic model, Bulletin of Mathematical Biology 60 (1998), no. 6, 1123-1148.

[14] H. L. Smith, Competitive coexistence in an oscillating chemostat, SIAM Journal on Applied Mathematics 40 (1981), no. 3, 498-522.

[15] S. Y. Tang and L. S. Chen, Density-dependent birth rate, birth pulses and their population dynamic consequences, Journal of Mathematical Biology 44 (2002), no. 2, 185-199.

Xinyu Song: Department of Mathematics, Xinyang Normal University, Xinyang, Henan 464000, Henan, China

E-mail address: xysong88@163.com

Zhong Zhao: Department of Mathematics, Huanghuai University, Xinyang,

Zhumadian 463000, Henan, China

E-mail address: zhaozhong8899@163.com 


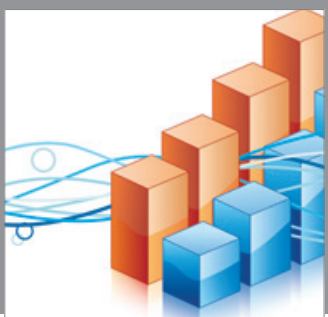

Advances in

Operations Research

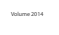

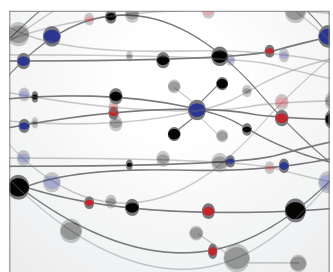

\section{The Scientific} World Journal
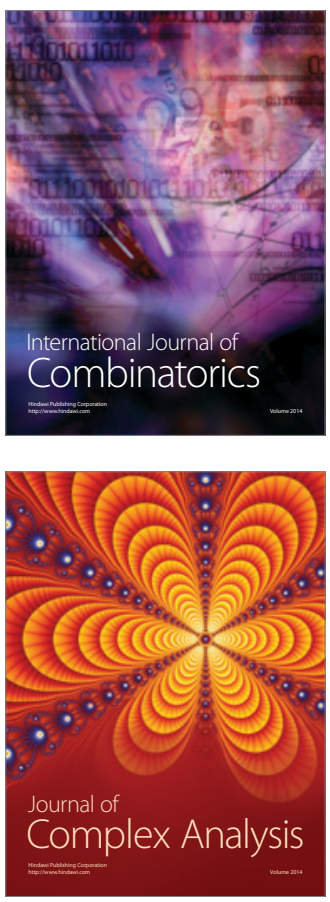

International Journal of

Mathematics and

Mathematical

Sciences
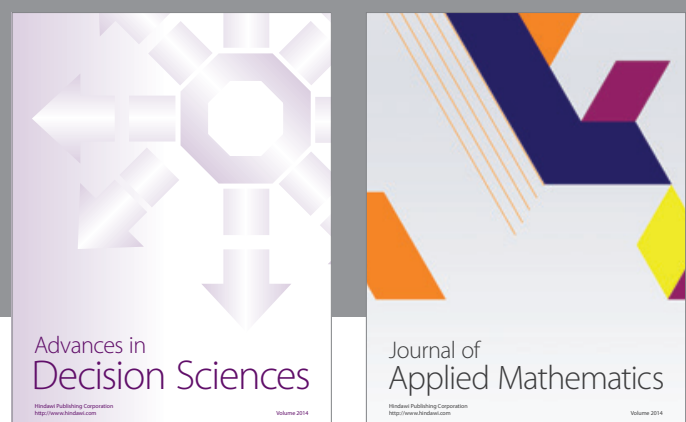

Journal of

Applied Mathematics
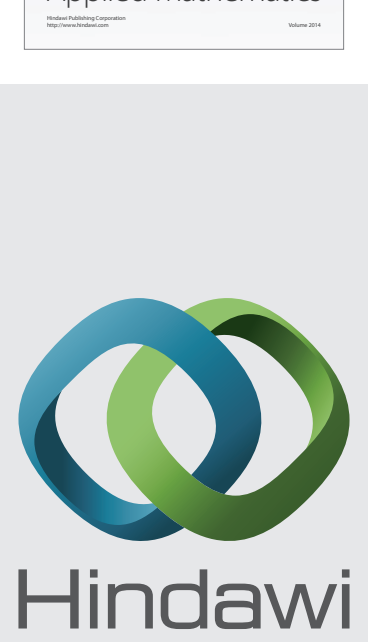

Submit your manuscripts at http://www.hindawi.com
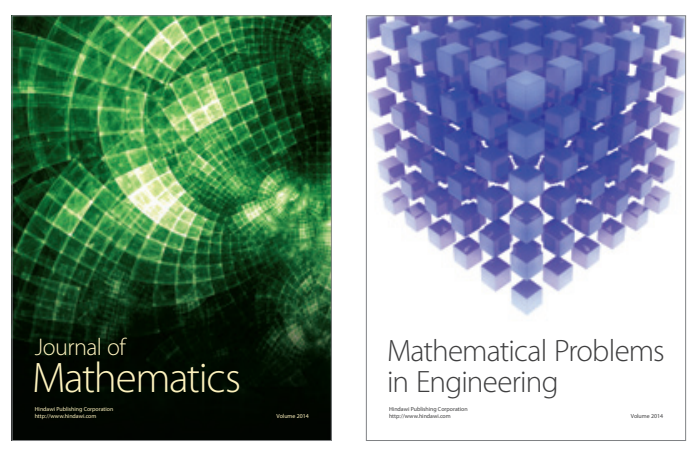

Mathematical Problems in Engineering
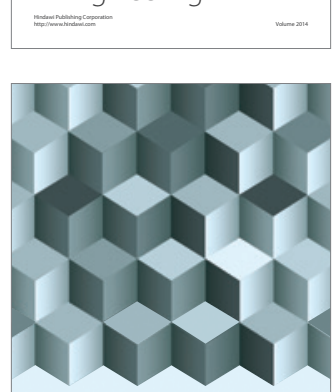

Journal of

Function Spaces
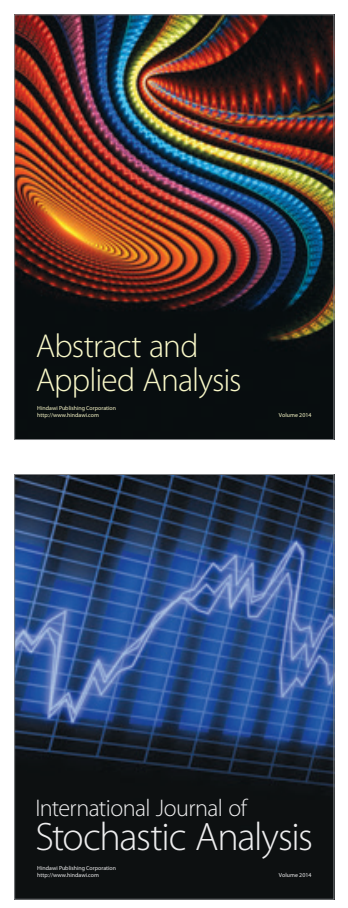

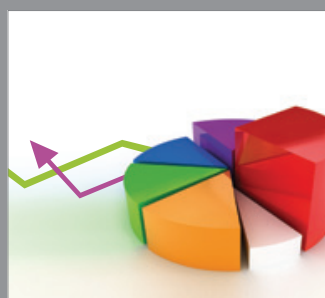

ournal of

Probability and Statistics

Promensencen
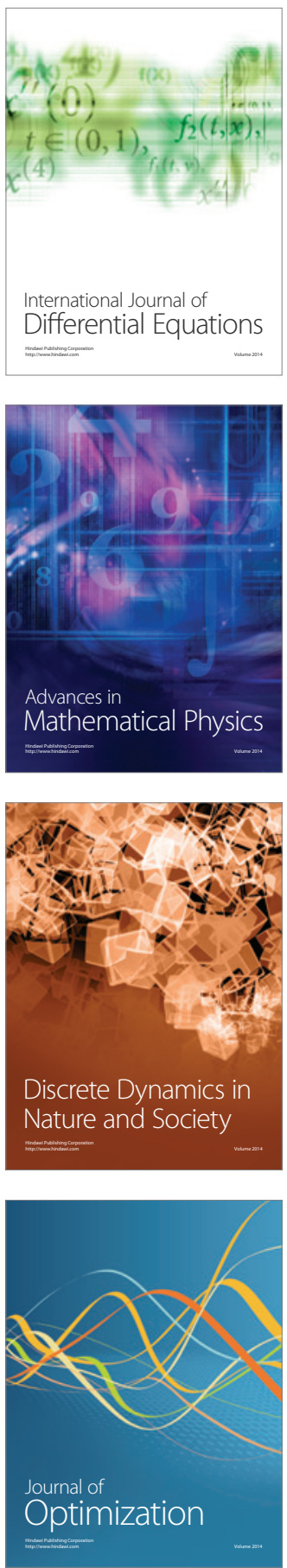